\title{
Long-Term Exposure to Residential Greenness and Neurodegenerative Disease Mortality Among the Elderly: A 13-Year Follow-Up Cohort Study
}

Lucia Rodriguez Loureiro ( $\square$ lucia.rodriguez.loureiro@vub.be )

Vrije Universiteit Brussel https://orcid.org/0000-0003-0092-3748

Sylvie Gadeyne

Vrije Universiteit Brussel

Mariska Bauwelinck

Vrije Universiteit Brussel

Wouter Lefebvre

VITO: VITO NV

Charlotte Vanpoucke

IRCEL-CELINE

Lidia Casas

University of Antwerp: Universiteit Antwerpen

\section{Research Article}

Keywords: Greenness, Mortality, Neurodegenerative disease, Dementia, Air pollution, Long-term exposure

Posted Date: February 7th, 2022

DOI: https://doi.org/10.21203/rs.3.rs-1324929/v1

License: (a) (i) This work is licensed under a Creative Commons Attribution 4.0 International License. Read Full License 


\section{Abstract \\ Background}

Living in greener areas is associated with slower cognitive decline and reduced dementia risk among the elderly, but the evidence with neurodegenerative disease mortality is scarce. We studied the association between residential surrounding greenness and neurodegenerative disease mortality in an elderly population.

\section{Methods}

We used data from the 2001 Belgian census linked to mortality register data during 2001-2014. We included individuals aged 60 years or older and residing in the five largest Belgian urban areas at baseline (2001). Exposure to residential surrounding greenness was assessed using the Normalized Difference Vegetation Index (NDVI) within 500-m from residence. We considered all neurodegenerative diseases and four specific outcomes: Alzheimer's disease, vascular dementia, unspecified dementia, and Parkinson's disease. We fitted Cox proportional hazard models to obtain hazard ratios (HR) and 95\% confidence intervals $(\mathrm{Cl})$ of the associations between one interquartile range (IQR) increment in surrounding greenness and neurodegenerative disease mortality outcomes. Furthermore, we conducted mediation analyses to assess potential mediation by air pollution $\left(\mathrm{PM}_{2.5}\right)$, and stratification analyses to explore effect modification by sociodemographic characteristics.

\section{Results}

From 1,134,502 individuals included at baseline, $6.1 \%$ died from neurodegenerative diseases during followup. After full adjustment, one IQR (0.22) increment of surrounding greenness was associated with a $4-5 \%$ reduction in premature mortality from neurodegenerative diseases, Alzheimer's disease, vascular and unspecified dementia [e.g., for Alzheimer's disease mortality: HR 0.95 (95\% Cl: 0.93, 0.98)]. No association was found with Parkinson's disease mortality. Reductions in air pollution concentrations could potentially mediate $58 \%(95 \% \mathrm{Cl}: 31.7 \%, 95.7 \%)$ of the observed effect with all neurodegenerative disease mortality. Associations were strongest in the lower educated and residents from most deprived neighbourhoods.

\section{Conclusions}

Living near greener spaces may reduce the risk of neurodegenerative disease mortality among the elderly, partly mediated by a reduction in air pollution concentrations. Socioeconomically disadvantaged groups may experience the greatest beneficial effect.

\section{Background}


Worldwide, the population is ageing, driving a dramatic increase in the burden of neurodegenerative diseases [1]. Moreover, population ageing is occurring faster in urban areas [2], which could contribute to

an exacerbated risk of neurodegenerative diseases following exposure to urban environmental hazards [35].

Exposure to green spaces may benefit urban dwellers by promoting healthy ageing [6] and reducing premature mortality [7]. Hypothesized mechanisms include inducing stress restoration, providing opportunities for physical activity and social cohesion, and mitigating environmental hazards [8]. However, the available evidence on the association between green spaces and neurodegenerative diseases among the elderly is mixed and inconclusive, and studies have so far primarily focused on dementia or its precursor, cognitive decline $[9,10,19-21,11-18]$. As far as we are aware, only one ecological and three longitudinal studies investigated the relationship between exposure to green spaces and neurodegenerative disease mortality: two longitudinal studies reported no association [22, 23], while the other two observed a beneficial association $[24,25]$. Of these, only two further used a specific outcome of neurodegenerative disease mortality, namely, dementia mortality [23, 24].

Among the suggested mechanisms underlying the beneficial effects of green spaces, mitigation of ambient air pollution may be important for neurodegenerative diseases, as it is estimated to account for $4 \%$ of the total risk of dementia in later life [26]. Still, the effect of air pollution on other neurodegenerative diseases remains unclear $[27,28]$.

Finally, the health-related effect of green spaces could differ across social strata, as individual and neighbourhood social factors may influence exposure and susceptibility to residential green spaces [29, $30]$. However, this has been scarcely addressed in relation to neurodegenerative diseases $[13,18]$.

In this study we examined the association between long-term exposure to green spaces and general and specific neurodegenerative disease mortality among the elderly (aged 60 years and older) residing in the five largest Belgian urban areas. Additionally, we assessed mediation by air pollution, and effect modification by social factors (gender and individual and neighbourhood socioeconomic position) in the studied associations.

\section{Methods}

\section{Study design and study population}

We used a longitudinal dataset based on a linkage between the 2001 Belgian census (baseline) and register data on emigration and mortality for the follow-up period from October 1, 2001, until December 31 , 2014. Furthermore, environmental data (i.e., green spaces and air pollution) were linked to this dataset using the residential address of each person at baseline. Our study population included all noninstitutionalized individuals aged 60 years and older, and officially residing in one of the five largest Belgian urban areas (Antwerp, Ghent, Brussels, Charleroi, and Liège). These areas were identified following the definition of urban area provided by Statistics Belgium (in Dutch: stadsgewest), consisting of the city 
and its respective commuting zone [31]. We included individuals with complete data on the residential address (observations excluded: $\mathrm{n}=11,824 ; 1.03 \%$ ).

\section{Neurodegenerative disease mortality}

Mortality data included the causes of death issued from the death certificates, using the codes from the 10th revision of the International Classification of Disease (ICD-10) [32]. We defined several outcomes of neurodegenerative disease mortality according to previous literature $[23,33]$. We considered deaths from all neurodegenerative diseases [including dementia (ICD-10: F00-F03); motor-neuron disease (ICD-10: G12.2); Parkinson's disease (ICD-10: G20-G22); Alzheimer's disease (ICD-10: G30); other neurodegenerative diseases of the nervous system, not elsewhere classified (ICD-10: G31); and multiple sclerosis (ICD-10: G35)] and four specific outcomes: Alzheimer's disease (AD) (G30); vascular dementia (F01); unspecified dementia (F03); and Parkinson's disease (PD) (G20-G22). Neurodegenerative diseases are often underreported in death certificates $[34,35]$. We therefore included all death certificates with any mention to the abovementioned outcomes (as an underlying, immediate, intermediate, or additional cause of death).

\section{Residential surrounding greenness}

Surrounding greenness around the residential address at baseline was measured using the Normalized Difference Vegetation Index (NDVI). A detailed description of the methodology used for the obtention of the indicator, as well as on the individual assignment of the exposure can be found in Bauwelinck et al. (2021). In brief, this metric captures vegetation density and is derived by applying the ratio between visible and near-infrared light bands to atmospherically corrected satellite images [37]. These were obtained for the 2006 summer period, with a $30 \mathrm{~m}$ resolution. Negative NDVI values representing water surfaces were set to zero prior to the calculation of the residential surrounding greenness indicator [36]. The index therefore ranged from 0 to 1 , corresponding to no green and maximum green density, respectively. We calculated the average index of surrounding greenness in three circular buffers at Euclidean distances of 300-m, 500-m, and 1,000-m from the residence.

\section{Ambient air pollution}

We considered air pollution as a potential mediator in the associations between residential surrounding greenness and neurodegenerative disease mortality. Data was obtained from the Belgian Interregional Environmental Agency (IRCEL-CELINE) (www.irceline.be). Air pollutant concentrations are constantly measured through a vast network of monitoring stations and then used in spatial-temporal (kriging) interpolation models. These data are further combined with Gaussian models including traffic and industrial sources and meteorological data to estimate air pollutant concentrations at high spatial resolution $(25 \mathrm{~m})$ in the whole Belgian territory $[38,39]$. We used 2005 annual average concentrations $\left(\mu \mathrm{g} / \mathrm{m}^{3}\right)$ of fine particulate matter $\left(\mathrm{PM}_{2.5}\right)$.

\section{Sociodemographic and socioeconomic characteristics}

Sociodemographic and socioeconomic characteristics at baseline were obtained from the 2001 Belgian census. Sociodemographic covariates included age [both as a continuous and with a 5-year 
categorization, from [60-65) until [95 and older)], gender, migrant background origin [Belgium, other highincome country (HIC), and low and middle-income country (LMIC)] and household living arrangement [cohabiting, single and other (e.g., multigenerational households)]. Individual socioeconomic position (SEP) was measured through attained educational level (tertiary, higher secondary, lower secondary, and lower and no education) and housing tenure (owner and tenant). Neighbourhood SEP was approximated using the median net taxable household income for the year 2005 at the level of the census tract (i.e., due to privacy reasons, the smallest geographical unit for which residential address is reported, comparable to neighbourhoods), available at Statistics Belgium (https://statbel.fgov.be/en), and unemployment rate in the census tract derived from the 2001 Belgian census.

\section{Statistical analyses}

We conducted multiple imputation to minimize potential selection bias introduced by missing information on any of the variables, observed in $19.6 \%$ of the study population. We used chained equations, carrying out 25 imputations with 10 iterations that generated 25 datasets. All the covariates were used in the process of multiple imputation. We further included as a predictor in the imputation process the NelsonAalen estimator of the cumulative hazard to the survival time for all neurodegenerative diseases [40].

We examined Spearman correlations between surrounding greenness, $\mathrm{PM}_{2.5}$, and neighbourhood SEP. We specified Cox proportional hazards models using age as underlying time scale. Hazard Ratios (HR) and corresponding $95 \%$ confidence intervals $(95 \% \mathrm{Cl})$ of the associations between residential surrounding greenness and neurodegenerative disease mortality. We a priori selected a 500-m buffer from residence for surrounding greenness, following the methodology used in previous studies [36, 41]. Observations were censored when the person died from other causes, emigrated from Belgium or end of the follow-up occurred. We included strata terms for each 5-year categorized age group and gender. We additionally specified a frailty term to account for the cluster effects of residing in one of the five largest urban areas. Our main model was built adjusting stepwise by covariates: Model 1 (M1) included the baseline hazard with the strata and the frailty terms; Model 2 (M2) was further adjusted by migrant background, household living arrangement, educational level, and housing tenure; and Model 3 (M3) added the median household income of the census tract of residence. The reported HRs were combined from the estimates obtained from the 25 imputed datasets according to Rubin's rules [42]. To examine the linearity of the exposureresponse relationship with surrounding greenness, we randomly selected one of the 25 imputed datasets and fitted natural splines with three degrees of freedom. We compared the improvement of goodness of fit with a likelihood ratio test (LRT) to the main model (M3). Only slight deviations from linearity were observed (Figure S1), hence we included surrounding greenness as a linear term into our models. Mortality associations were reported by one interquartile range (IQR) increment in surrounding greenness.

In additional analyses we investigated the role of air pollution in the studied associations [43]. We firstly further adjusted our main models (M3) for $\mathrm{PM}_{2.5}$ concentrations. Next, we evaluated potential mediation by $\mathrm{PM}_{2.5}$ in the associations between surrounding greenness and neurodegenerative disease mortality outcomes that were significant in our main models. We used the package mediation [44] in R statistical software to obtain the average causal mediation effects (ACME), also known as indirect effects, and the 
average direct effects (ADE) in each imputed dataset $(n=25)$. This allows to calculate the proportion of the total effect mediated by air pollution dividing the ACME by the total effect [ACME/(ACME + ADE)]. Here it can be interpreted as the proportion of risk reduction of neurodegenerative disease mortality after exposure to surrounding greenness explained by a reduction in $\mathrm{PM}_{2.5}$ concentrations [45]. We computed the average of the estimates and the corresponding 95\% Quasi Bayesian confidence intervals $(95 \% \mathrm{Cl})$, based on 1,000 Monte Carlo simulations, obtained from the 25 imputed datasets.

To determine if the observed effect in our main models was modified by sociodemographic characteristics, we stratified our models by gender, educational level, and neighbourhood SEP (by quartiles of the median net taxable income in the census tract of residence).

Finally, to assess the robustness of our main models, we conducted several sensitivity analyses of our main models: (1) using surrounding greenness within 500-m categorized into quintiles; (2) using the 300-m and 1,000-m buffer sizes of residential surrounding greenness; (3) using perceived neighbourhood greenness as an alternative indicator of exposure to green spaces, following the methodology from a previous study [30]; (4) adjusting the main models for the unemployment rate in the census tract as an alternative indicator of neighbourhood SEP; (5) limiting our analyses to specific population groups: (a) the complete case population (i.e., individuals with no missing data on the covariates); (b) non-movers, i.e., individuals who resided in the same census tract between 1991 and 2001 (10 years prior to baseline); (c) individuals originating from Belgium; (d) individuals younger than 80 years old at baseline; and (e) individuals residing in the city, leaving out commuting zone residents.

All statistical analyses were conducted in R/4.0 [46], using the packages mice [47], survival[48], splines [46] and ggplot2 [49] and dependencies.

\section{Results}

Our total (imputed) study population consisted of 1,134,502 individuals aged 60 years or older and residing in the five largest Belgian urban areas in 2001 (Table 1). During follow-up (2001-2014), 69,149 individuals (6.1\%) died from neurodegenerative diseases as any cause of death, 21,039 (1.9\%) died from Alzheimer's disease (AD), and 31,302 (2.8\%) died from unspecified dementia. Fewer individuals died from vascular dementia or Parkinson's disease (PD) ( $0.5 \%$ and $0.9 \%$, respectively). The average age at baseline was 72 years $(S D \pm 7.7)$. The population was mainly composed by women, residents originating from Belgium, individuals with primary or no formal education, and cohabiting with their partner. The median exposure to residential surrounding greenness (buffer size: 500-m) was 0.61 (IQR: 0.22 ). In the complete case population $(n=911,648)$, deaths from these diseases were slightly lower (e.g., death from all neurodegenerative diseases: $n=52,780,5.8 \%$, while environmental exposures were similar. A detailed description of all environmental variables in both populations is shown in Table S1. 
Table 1

Neurodegenerative disease mortality (2001-2014) and baseline characteristics in the full imputed and complete case population.

Neurodegenerative disease mortality, n (\%)

All neurodegenerative diseases

Alzheimer's disease

Vascular dementia

Unspecified dementia

Parkinson's disease

Age at baseline, mean \pm SD

Women, $\mathrm{n}(\%)$

Migrant background, $\mathrm{n}(\%)$

Belgian

Other HIC

LMIC

Educational level, n (\%)

Tertiary

Higher Secondary

Lower Secondary

Low/Primary

Housing tenure, $\mathrm{n}(\%)$

Owner

Tenant

Household living arrangement, $\mathbf{n}(\%)$

Cohabiting

Single

Other

Median net taxable income $(€)$, median (IQR)

Non-movers (1991-2001), n (\%)
Full (imputed) population $(n=1134502)$

\begin{tabular}{ll}
$69149(6.10)$ & $52780(5.79)$ \\
\hline $21039(1.85)$ & $16161(1.77)$ \\
$5651(0.50)$ & $4204(0.46)$ \\
$31302(2.76)$ & $23570(2.59)$ \\
$10054(0.89)$ & $7932(0.87)$ \\
$71.8 \pm 7.7$ & $71.5 \pm 7.6$ \\
$644687(56.8)$ & $514121(56.4)$
\end{tabular}

1001938 (88.3)

$94811(8.4)$

$37753(3.3)$

Complete case population $(n=911648)$

52780 (5.79)

16161 (1.77)

4204 (0.46)

23570 (2.59)

813136 (89.2)

70497 (7.7)

28015 (3.1)

143927 (12.7)

118543 (13.0)

182750 (16.1)

150398 (16.5)

299778 (26.4)

242632 (26.6)

508047 (44.8)

400075 (43.9)

859624 (75.8)

700281 (76.8)

274878 (24.2)

211367 (23.2)

715546 (63.1)

591646 (64.9)

389895 (34.4)

298746 (32.8)

29061 (2.6)

21256 (2.3)

$19094(4,620)$

$19206(4,530)$

1003052 (88.4)

810682 (88.9) 
Full (imputed) population

$(n=1134502)$

\section{Urban area, $\mathbf{n}(\%)$}

\begin{tabular}{lll} 
Antwerp & $248391(21.9)$ & $207726(22.8)$ \\
\hline Ghent & $120809(10.6)$ & $101904(11.2)$ \\
\hline Brussels & $505297(44.5)$ & $403091(44.2)$ \\
\hline Charleroi & $104774(9.2)$ & $78782(8.6)$ \\
\hline Liège & $155231(13.7)$ & $120145(13.2)$ \\
$\begin{array}{l}\text { Residential surrounding greenness (NDVI, } \\
\mathbf{5 0 0 - m ) , ~ m e d i a n ~ ( I Q R ) ~}\end{array}$ & $0.61(0.22)$ & $0.61(0.22)$ \\
\hline $\begin{array}{l}\text { Ambient air pollution [PM } \\
\text { median (IQR) }\end{array}$ & $19.05(2.09)$ & $19.07(2.09)$ \\
\hline
\end{tabular}

Note: SD, Standard Deviation; IQR, interquartile range; $€$, euro currency; NDVI, Normalized Difference Vegetation Index; $\mathrm{PM}_{2.5}$, Particulate Matter with an aerodynamic diameter $<2.5 \mu \mathrm{m}$.

Five largest Belgian urban areas, 2001-2014.
Complete case population $(n=911648)$

[Insert Table 1 (at the bottom of the file) here]

Spearman correlations are displayed in Figure S2. We observed moderate negative correlations between surrounding greenness and $\mathrm{PM}_{2.5}$ concentrations (e.g., for surrounding greenness within $500-\mathrm{m}: \mathrm{r}=-0.58$ ). Neighbourhood SEP (median income) was moderately correlated with surrounding greenness ( $r=0.50$ for all buffer sizes), and weakly negatively correlated with air pollution ( $r=-0.23)$.

Surrounding greenness associations generally attenuated with increasing covariate adjustment in our stepwise models. Associations in fully adjusted models (M3) showed a reduction of $4 \%$ in the risk of all neurodegenerative disease mortality, and of $5 \%$ in the risk of $A D$, vascular dementia and unspecified dementia mortality (Figure 1 and Table S2). Contrarily, for PD mortality, we observed a non-significant, almost null association.

When further adjusting our main models for $\mathrm{PM}_{2.5}$ concentrations (Table 2) we generally observed an attenuation in the association between surrounding greenness and all outcomes

of neurodegenerative disease mortality, although became null for unspecified dementia and strengthened for vascular dementia mortality. Results from the mediation analyses by $\mathrm{PM}_{2.5}$ are reported in Table S3. Assuming that all statistical assumptions to conduct mediation were met [50], our findings suggest that the average proportion of the effect of surrounding greenness on neurodegenerative disease mortality mediated by $\mathrm{PM}_{2.5}$ concentrations was $58.0 \%$ (95\% Cl: $31.7 \%$, 95.7\%). The same pattern for mediation 
results was observed for $A D$ and unspecified dementia mortality, but not for vascular dementia mortality. Moreover, for these specific outcomes, none of the estimates for mediation were statistically significant.

Table 2

Associations (HRs) and 95\% Cl between surrounding greenness and neurodegenerative disease mortality after adjustment for $\mathrm{PM}_{2.5}$.

\begin{tabular}{|c|c|c|c|c|c|}
\hline & $\begin{array}{l}\text { Neurodegene- } \\
\text { rative disease } \\
\text { mortality }\end{array}$ & $\begin{array}{l}\text { Alzheimer's } \\
\text { disease } \\
\text { mortality }\end{array}$ & $\begin{array}{l}\text { Vascular } \\
\text { dementia } \\
\text { mortality }\end{array}$ & $\begin{array}{l}\text { Unspecified } \\
\text { dementia } \\
\text { mortality }\end{array}$ & $\begin{array}{l}\text { Parkinson's } \\
\text { disease } \\
\text { mortality }\end{array}$ \\
\hline & $\mathrm{HR}(95 \% \mathrm{Cl})$ & $\mathrm{HR}(95 \% \mathrm{Cl})$ & $\mathrm{HR}(95 \% \mathrm{Cl})$ & $\mathrm{HR}(95 \% \mathrm{Cl})$ & $\mathrm{HR}(95 \% \mathrm{Cl})$ \\
\hline \multirow{2}{*}{$\begin{array}{l}\text { Main model } \\
\text { (M3) }\end{array}$} & $0.96(0.95$ & $0.95(0.93$ & $0.95(0.90$ & $0.95(0.93$ & $1.01(0.97$ \\
\hline & $0.97)$ & $0.98)$ & $1.00)$ & $0.97)$ & 1.05) \\
\hline $\begin{array}{l}\text { Main model } \\
\text { (M3) + } \mathrm{PM}_{2.5}\end{array}$ & & $0.97(0.93$ & $0.93(0.87$ & $1.00(0.97$ & $0.99(0.95$ \\
\hline$\left(\mu \mathrm{g} / \mathrm{m}^{3}\right)$ & 1.00) & $1.00)$ & $0.99)$ & 1.03) & 1.04) \\
\hline \multicolumn{6}{|c|}{$\begin{array}{l}\text { Abbreviations: } \mathrm{HR} \text {, Hazard Ratio; } 95 \% \mathrm{Cl}, 95 \% \text { confidence intervals; } \mathrm{PM}_{2.5}\left(\mu \mathrm{g} / \mathrm{m}^{3}\right) \text {, particulate matter } \\
\text { with an aerodynamic diameter }<2.5 \mu \mathrm{m} \text {, measured in micrograms per cubic metre. }\end{array}$} \\
\hline \multicolumn{6}{|c|}{ Five largest Belgian urban areas, 2001-2014. } \\
\hline \multicolumn{6}{|c|}{$\begin{array}{l}\text { Cox regression models using age as the underlying time scale for the follow-up period October } 1,2001 \\
\text { December } 31,2014 \text {. Main model (M3) stratified by gender and } 5 \text {-year age groups, adjusted by migrant } \\
\text { background, household living arrangement, educational level, housing tenure, median net taxable } \\
\text { income at the level of the statistical sector. and includina a frailty term for the urban areas. }\end{array}$} \\
\hline
\end{tabular}

The results of the stratified analyses by gender, educational level, and neighbourhood SEP are shown in Figure 2 and are fully reported in Table S4. We found almost no differences between men and women. We did not observe a clear pattern across educational levels. However, individuals with primary or no formal education showed consistently a stronger beneficial effect of surrounding greenness for all neurodegenerative disease, AD, and unspecified dementia mortality [e.g., for AD: HR 0.93 (95\%Cl: 0.90, 0.97)]. By neighbourhood SEP, we observed that for all neurodegenerative disease and unspecified dementia mortality the beneficial effect of surrounding greenness was strongest in individuals living in the most deprived (Q1 and Q2) neighbourhoods [e.g., for unspecified dementia: HR 0.93 (95\%Cl: 0.90, 0.96), HR 0.91 (95\%Cl: 0.88, 0.96); respectively]. Contrarily, for AD mortality this was observed in residents from the least deprived (Q4) neighbourhoods [HR 0.91 (95\%Cl: 0.84, 0.98)]. For vascular dementia mortality significant beneficial associations were observed in individuals residing in the second least deprived (Q3) and the most deprived (Q1) neighbourhoods.

The results of the sensitivity analyses were overall consistent with our main findings shown in Figure 1 (Table S5-S6). Using the surrounding greenness indicator categorized into quintiles we observed the strongest associations for all neurodegenerative outcomes (except PD) for the greenest quintile compared 
to the least green quintile. The results using different buffer sizes were very similar. Using perceived neighbourhood greenness as alternative exposure indicator yielded null associations with AD and vascular dementia mortality, whereas with unspecified dementia it was stronger (HR $0.93,95 \% \mathrm{Cl}: 0.91,0.94)$. The associations with $A D$ mortality lost significance after alternatively adjusting for unemployment rate in the census tract; in contrast, the inverse associations with vascular and unspecified dementia mortality were stronger. Using the complete case population and including only the population groups aged $60-80$ and living in the city centre generally attenuated the associations, yielding in some cases a borderline nonsignificant association. Limiting the analyses to non-movers and residents originating from Belgium strengthened the observed associations. Finally, we observed an inverse but non-significant association with PD mortality when excluding individuals living in the commuting zone (HR 0.96, 95\% Cl: 0.91, 1.02).

\section{Discussion}

Our findings suggest that exposure to urban residential greenness reduces mortality from all neurodegenerative diseases, and specifically from Alzheimer's disease (AD) and dementia. We found no significant associations with Parkinson's disease (PD) mortality. The observed beneficial effects were potentially mediated by a reduction in air pollution concentrations. Moreover, we found that this protective effect was generally stronger in individuals with lower education. We also found the strongest beneficial associations for overall neurodegenerative diseases and unspecified dementia mortality in individuals residing in the most deprived neighbourhoods. In contrast, for AD mortality, the strongest beneficial association was observed in wealthier neighbourhoods.

Our results regarding reduced premature mortality from neurodegenerative diseases with increased surrounding greenness are comparable to those reported by Klompmaker et al. (2021) in a study over 10 million adults (aged $\geq 30$ years). Using the same classification of neurodegenerative diseases, the authors observed a $2 \%$ reduction (per 0.14 increase) in neurodegenerative disease mortality with surrounding greenness. Stratified by age, results were similar in the elderly ( $\geq 65$ years) [24]. An ecological study in Greece also found a significant inverse association (HR 0.91) between greenness and mortality from diseases of the nervous system (ICD-10 codes G00-G99) [25]. Two longitudinal studies found a protective but non-significant association with neurodegenerative disease mortality [22, 23]. James et al. (2016) included 108,630 elderly female nurses followed between $2000-2008$, yielding an HR of 0.93 per 0.1 increase in surrounding greenness. Klompmaker et al. (2020) used survey data of 339,633 individuals linked with mortality data (2003-2007), and showed a similar effect estimate as Klompmaker et al. (2021), but with wider confidence intervals. Potentially these studies did not find significant associations because of an insufficient statistical power due to smaller study populations combined with shorter mortality follow-up periods.

Our study findings were consistent for different dementia subtypes. Two of the abovementioned studies additionally assessed dementia mortality (all dementia types, ICD-10 codes: F00-F03). Klompmaker et al. (2021) showed a significant $4 \%$ reduction in the risk of dementia mortality with increased surrounding greenness. Klompmaker et al. (2020) reported a similar but non-significant effect. Other longitudinal 
studies using health administrative databases found a beneficial association between green spaces and an outcome including all dementia types and AD (ICD-10 codes: F00-F03, G30, respectively) [16, 17]. However, studies evaluating the effect of green spaces on $A D$ alone have shown contradictory findings [18-21]. Moreover, we are not aware of prior studies evaluating the association between green spaces and vascular dementia. Thus, further research is needed to confirm our results.

Suggested mechanisms underlying the direct beneficial effect of exposure to green spaces on neurodegenerative disease mortality include inducing psychological restoration and reducing stress [8], potentially preventing depression, a risk factor for dementia [51]. Additionally, there is suggestive evidence that green spaces could increase physical activity in the elderly [6], reducing the risk of dementia and AD [52]. Social isolation is moreover associated with an increased risk of cognitive decline and dementia [53], and greener neighbourhoods could enhance social cohesion and mitigate feelings of loneliness in older adults $[54,55]$. Finally, green spaces could contribute to the mitigation of environmental hazards, including air pollution [56]. Our findings suggest that the associations between residential greenness and neurodegenerative disease mortality are partly mediated by a reduction in air pollution. We only reported results with $\mathrm{PM}_{2.5}$. In alternative models with $\mathrm{NO}_{2}$ (results not shown), we observed similar findings. So far, only one longitudinal study on green spaces and cognitive function explored potential mediation by air pollution, but no evidence of mediation effects was found [13]. Current evidence establishes a strong link between air pollution and $A D$ and dementia [26]. The filtering effect of green spaces removing pollutants from the atmosphere has been proven to be generally small $[57,58]$. However, green spaces could decrease temperature in cities [59], indirectly improving air quality by reducing the generation, transportation and toxicity of pollutants [60]. Likewise, fewer sources of air pollution are found in greener areas [43]. Noise pollution or proximity to roads have also been associated with an increased risk of dementia independently from air pollution levels $[3,4]$, but we unfortunately lacked such information.

As part of the sensitivity analyses, we used perceived neighbourhood greenness as alternative exposure indicator, where we found no association with $A D$ nor vascular dementia mortality and a stronger association with unspecified dementia compared to the results of our main models using surrounding greenness. We suspect that perceived neighbourhood greenness may partly capture certain aspects of neighbourhood socioeconomic position (SEP). As such, this model may be overadjusted for SEP. Our findings are probably explained by individuals with high SEP having a higher likelihood of getting a record of dementia aetiology in death certificates [61], and, moreover, residing more often in areas where a higher proportion of individuals report very good neighbourhood greenspace provision.

No beneficial association between greenness and PD mortality was found, probably a result of the differing aetiology of PD compared to that of the other neurodegenerative diseases under study [62]. Additionally, in sensitivity analysis the associations with PD changed direction and became beneficial when commuting zone residents were excluded. Morphologically, these areas are characterized by an extensive land use in both housing and commercial activities [31]. Thus, we may speculate that increased risk of PD mortality could partly be explained by exposure to agricultural land, potentially encompassing exposure to pesticides, although this has been mainly explored for agricultural workers [62], being the 
evidence available for residential exposures currently limited [63]. Furthermore, we were not able to further explore this hypothesis since we lacked data on different types of green such as agricultural land.

Comparison between different population subgroups in the stratified analyses should be done with caution, given statistical restrictions in such interpretations. We observed strongest beneficial effects of living near greener areas in the lower educated for all neurodegenerative diseases and AD mortality. No clear patterns were found for other studied causes. Similarly, the longitudinal study of de Keijzer et al. (2018) did not find consistent evidence for differences between different education groups in the association with residential greenness and cognitive decline. Regarding neighbourhood SEP, we observed a general trend for all neurodegenerative disease and dementia mortality, where strongest associations with residential greenness were found in more socially deprived areas. The aforementioned study by de Keijzer et al. (2018) observed similar patterns with cognitive decline. Lower SEP has been linked to both poorer living conditions and limited access to resources which may be related to increased risk of cognitive decline in later life [64]. Additionally, availability of residential green has been associated to reduced risk of mortality, where health benefits seemed to be largest among most deprived population groups [65]. Such gradient was partly confirmed by our study results, although for AD mortality we found strongest beneficial associations in individuals residing in wealthier areas. This contradicts the findings from Brown et al. (2018), in which a trend by neighbourhood SEP in the association between greenness and AD prevalence was found, showing the strongest estimate in low-income neighbourhoods.

Our study comes with several limitations. The major limitation of our study is that we were not able to control for lifestyle factors, e.g., body mass index (BMI), smoking status and alcohol consumption, which are well-known risk factors for AD and dementia [26]. Prior studies that were able to account for these factors observed a small attenuation in the association [22, 23]. However, comparability of findings after such adjustment is challenging given several important differences in study design, population characteristics and exposure assessment. Hence, the direction of potential bias in our effect estimates remains unclear. Furthermore, our study did not include time-varying variables of exposure throughout the follow-up period. We only had one measure of surrounding greenness for the year 2006, close to the middle of the follow-up period, which is another limitation of our study. We assumed that, although the quantity of green spaces may vary across time, their spatial distribution remains relatively stable. However, no other exposure information was available for other years to test this. Moreover, exposure assessment was based on the geocoded residential address at baseline (2001), and we lacked information on residential mobility during follow-up (2001-2014). Still, we were able to limit the analyses to a group of residents who did not move in the last 10 years prior to baseline, which did not invalidate our main results. Surrounding greenness captured all types and sizes of green spaces, independently of these being private or public. Also, limiting greenspace exposure assessment to the residence may result in exposure misclassification, as it potentially ignores exposure in other life spheres, e.g., working place. Similarly, episodes of nature interaction were not measured in quality (e.g., accessibility, type of use) nor in time (e.g., frequency, duration). We also relied on baseline information of sociodemographic characteristics, but these may not vary considerably among the elderly population. Applied missing values techniques present limitations given that the missing values may not be completely at random, which potentially affects

Page $12 / 22$ 
generalizability of our findings. However, by using two approaches to handle missing data (i.e., multiple imputation and listwise deletion), we may assume that main conclusions are fairly robust. Lastly, we excluded the institutionalized population (i.e., care homes) to minimise selection bias, given that almost half of individuals diagnosed with neurodegenerative diseases live in institutions [66].

Notwithstanding the limitations described, our study counts with an important number of strengths. We analysed the association between surrounding greenness and neurodegenerative diseases over a longer follow-up period (13.25 years) than prior studies [22-24]. We also relied on a high resolution environmental database, linked to the geocoded residential address of each individual officially residing in the five largest Belgian urban areas at baseline [36]. Our study was also the first to conduct mediation analyses in the studied associations by air pollution concentrations using individually linked exposure data. Finally, using large administrative data allowed us to study effect modification by gender and socioeconomic characteristics through stratification for representative subgroups.

\section{Conclusions}

In a large population-based cohort of elderly individuals, we observed a reduced risk of overall and specific neurodegenerative disease mortality (except for Parkinson's disease) associated to exposure to residential surrounding greenness. We also found that these associations are potentially mediated by reductions in air pollution concentrations. Additionally, the beneficial effect of exposure to green spaces on neurodegenerative disease mortality might be stronger in lower educated groups and individuals residing in more deprived neighbourhoods. In Europe, neurological disorders represent the third leading cause of death and disability, after cardiovascular diseases and cancer [67]. Still, up to $40 \%$ of dementia cases can be potentially prevented (WHO 2021). Our results highlight the importance of the living environment to promote healthy ageing and reduce the burden of neurodegenerative diseases, especially among the most vulnerable populations. Future research is needed to confirm our findings in other settings and to explore other underlying mechanisms linking surrounding greenness with neurodegenerative diseases.

\section{List Of Abbreviations}

NDVI, Normalised Difference Vegetation Index; IQR, interquartile range; HR, hazard ratio; $95 \% \mathrm{Cl}, 95 \%$ confidence interval; $\mathrm{PM}_{2.5}$, particulate matter with an aerodynamic matter smaller than $2.5 \mu \mathrm{m} ; \mathrm{AD}$, Alzheimer's disease; PD, Parkinson's disease; SEP, socioeconomic position.

\section{Declarations}

\section{Ethics approval and consent to participate}

Not applicable.

\section{Consent for publication}

Not applicable. 


\section{Availability of data and materials}

The data that support the findings of this study includes identifying information on participants and was used under license for the current study, and hence not publicly available. Data codebooks and syntaxes used for the statistical analyses are however available from the authors upon request.

\section{Competing interests}

The authors declare that they have no competing interests.

\section{Funding}

Lucía Rodríguez Loureiro is funded by the Brussels Institute for Research and Innovation (INNOVIRIS) [project number: 2019-ANTICIPATE-26100]. Mariska Bauwelinck is funded by an individual PhD grant supported by the Research Foundation-Flanders (FWO) [grant number 11A9718N].

\section{Authors' contributions}

Lucía Rodríguez Loureiro: Conceptualization, Methodology, Formal Analysis, Visualization, Writing-Original Draft, Writing-Review \& Editing; Sylvie Gadeyne: Conceptualization, Writing-Review \& Editing, Supervision, Funding acquisition; Mariska Bauwelinck: Resources, Writing-Review \& Editing; Wouter Lefebvre: Resources; Charlotte Vanpoucke: Resources; Lidia Casas: Conceptualization, Writing-Review \& Editing, Supervision, Funding acquisition. All authors read and approved the final manuscript.

\section{Acknowledgements}

We would like to acknowledge the important role of Statbel in this study (Directorate-general Statistics Statistics Belgium) for geocoding the census data and facilitating data linkages. We would also like to thank IRCEL-CELINE (Belgian Interregional Environment Agency) for providing the air pollution data. We furthermore thank Dr Tim Nawrot, from the University of Hasselt and the KULeuven, for his insightful comments of preliminary findings and useful suggestions for the development of the study objectives. The resources and services used in this work were provided by the VSC (Flemish Supercomputer Centre), funded by the Research Foundation - Flanders (FWO) and the Flemish Government.

\section{References}

1. GBD 2016 Neurology Collaborators. Global, regional, and national burden of neurological disorders, 1990-2016: a systematic analysis for the Global Burden of Disease Study 2016. Lancet Neurol. 2019;18:459-80. https://doi.org/10.1016/S1474-4422(18)30499-X.

2. United Nations. World Population Ageing [Online]. In: Econ. Soc. Aff. 2015. Available from: https://www.un.org/en/development/desa/population/publications/pdf/ageing/WPA2015_Report.pdf (Accessed 03.02.2022). 
3. Chen H, Kwong JC, Copes R, Tu K, Villeneuve PJ, van Donkelaar A, et al. Living near major roads and the incidence of dementia, Parkinson's disease, and multiple sclerosis: a population-based cohort study. Lancet. 2017;389:718-26. https://doi.org/10.1016/S0140-6736(16)32399-6.

4. Tzivian L, Dlugaj M, Winkler A, Weinmayr G, Hennig F, Fuks KB, et al. Long-Term Air Pollution and Traffic Noise Exposures and Mild Cognitive Impairment in Older Adults: A Cross-Sectional Analysis of the Heinz Nixdorf Recall Study. Environ Health Perspect. 2016;124:1361-8. https://doi.org/10.1289/ehp.1509824.

5. Weuve J, Bennett EE, Ranker L, Gianattasio KZ, Pedde M, Adar SD, et al. Exposure to Air Pollution in Relation to Risk of Dementia and Related Outcomes: An Updated Systematic Review of the Epidemiological Literature. Environ Health Perspect. 2021;129:96001. https://doi.org/10.1289/EHP8716.

6. de Keijzer C, Bauwelinck M, Dadvand P. Long-Term Exposure to Residential Greenspace and Healthy Ageing: a Systematic Review. Curr Environ Heal Reports. 2020;7:65-88. https://doi.org/10.1007/s40572-020-00264-7.

7. Rojas-Rueda D, Nieuwenhuijsen MJ, Gascon M, Perez-Leon D, Mudu P. Green spaces and mortality: a systematic review and meta-analysis of cohort studies. Lancet Planet Heal. 2019;3:e469-77. http://dx.doi.org/10.1016/S2542-5196(19)30215-3.

8. Nieuwenhuijsen MJ, Khreis H, Triguero-Mas M, Gascon M, Dadvand P. Fifty Shades of Green. Pathway to Healthy Urban Living. Epidemiology. 2017;28:63-71.

9. Clarke PJ, Ailshire JA, House JS, Morenoff JD, King K, Melendez R, et al. Cognitive function in the community setting: the neighbourhood as a source of 'cognitive reserve'? J Epidemiol Community Health. 2012;66:730. https://doi.org/10.1136/jech.2010.128116.

10. Wu Y-T, Prina AM, Jones AP, Barnes LE, Matthews FE, Brayne C, et al. Community environment, cognitive impairment and dementia in later life: results from the Cognitive Function and Ageing Study. Age Ageing. 2015;44:1005-11. https://doi.org/10.1093/ageing/afv137.

11. Wu Y-T, Prina AM, Jones A, Matthews FE, Brayne C. The Built Environment and Cognitive Disorders: Results From the Cognitive Function and Ageing Study II. Am J Prev Med. 2017;53:25-32. https://doi.org/10.1016/j.amepre.2016.11.020.

12. Zijlema WL, Triguero-Mas M, Smith G, Cirach M, Martinez D, Dadvand P, et al. The relationship between natural outdoor environments and cognitive functioning and its mediators. Environ Res. 2017;155:268-75. https://doi.org/10.1016/j.envres.2017.02.017.

13. de Keijzer C, Tonne C, Basagaña X, Valentín A, Singh-Manoux A, Alonso J, et al. Residential Surrounding Greenness and Cognitive Decline: A 10-Year Follow-up of the Whitehall II Cohort. Environ Health Perspect. 2018;126:77003. https://doi.org/10.1289/EHP2875.

14. Cherrie MPC, Shortt NK, Mitchell RJ, Taylor AM, Redmond P, Thompson CW, et al. Green space and cognitive ageing: A retrospective life course analysis in the Lothian Birth Cohort 1936. Soc Sci Med. 2018;196:56-65. https://doi.org/10.1016/j.socscimed.2017.10.038. 
15. Hystad P, Payette Y, Noisel N, Boileau C. Green space associations with mental health and cognitive function: Results from the Quebec CARTaGENE cohort. Environ Epidemiol. 2019;3:e040-0. https://doi.org/10.1097/EE9.000000000000004.

16. Paul LA, Hystad P, Burnett RT, Kwong JC, Crouse DL, van Donkelaar A, et al. Urban green space and the risks of dementia and stroke. Environ Res. 2020;186:109520.

https://doi.org/10.1016/j.envres.2020.109520.

17. Astell-Burt T, Navakatikyan MA, Feng X. Urban green space, tree canopy and 11-year risk of dementia in a cohort of 109,688 Australians. Environ Int. 2020;145:106102. https://doi.org/10.1016/j.envint.2020.106102.

18. Brown SC, Perrino T, Lombard J, Wang K, Toro M, Rundek T, et al. Health Disparities in the Relationship of Neighborhood Greenness to Mental Health Outcomes in 249,405 U.S. Medicare Beneficiaries. Int J Environ Res Public Heal. 2018;15:3. https://doi.org/10.3390/ijerph15030430.

19. Yuchi W, Sbihi H, Davies H, Tamburic L, Brauer M. Road proximity, air pollution, noise, green space and neurologic disease incidence: a population-based cohort study. Environ Heal. 2020;19:8. https://doi.org/10.1186/s12940-020-0565-4.

20. Liu C-C, Li C-Y, Kung S-F, Kuo H-W, Huang N-C, Sun Y, et al. Association of Environmental Features and the Risk of Alzheimer's Dementia in Older Adults: A Nationwide Longitudinal Case-Control Study. Int J Environ Res Public Heal. 2019;16:16. https://doi.org/ 10.3390/ijerph16162828.

21. Aitken WW, Lombard J, Wang K, Toro M, Byrne M, Nardi MI, et al. Relationship of Neighborhood Greenness to Alzheimer's Disease and Non-Alzheimer's Dementia Among 249,405 U.S. Medicare Beneficiaries. J Alzheimer's Dis IOS Press. 2021;81:597-606.

22. James P, Hart JE, Banay RF, Laden F. Exposure to Greenness and Mortality in a Nationwide Prospective Cohort Study of Women. Environ Health Perspect. 2016;124:1344-52. https://doi.org/10.1289/ehp.1510363.

23. Klompmaker JO, Hoek G, Bloemsma LD, Marra M, Wijga AH, van den Brink C, et al. Surrounding green, air pollution, traffic noise exposure and non-accidental and cause-specific mortality. Environ Int. 2020;134:105341. https://doi.org/10.1016/j.envint.2019.105341.

24. Klompmaker JO, Janssen NAH, Bloemsma LD, Marra M, Lebret E, Gehring U, et al. Effects of exposure to surrounding green, air pollution and traffic noise with non-accidental and cause-specific mortality in the Dutch national cohort. Environ Heal. 2021;20:82. https://doi.org/10.1186/s12940-021-00769-0.

25. Kasdagli M-I, Katsouyanni K, de Hoogh K, Lagiou P, Samoli E. Investigating the association between long-term exposure to air pollution and greenness with mortality from neurological, cardio-metabolic and chronic obstructive pulmonary diseases in Greece. Environ Pollut. 2022;292:118372. https://doi.org/10.1016/j.envpol.2021.118372.

26. Livingston G, Huntley J, Sommerlad A, Ames D, Ballard C, Banerjee S, et al. Dementia prevention, intervention, and care: 2020 report of the Lancet Commission. Lancet. 2020;396:413-46. https://doi.org/10.1016/S0140-6736(20)30367-6. 
27. Kasdagli M-I, Katsouyanni K, Dimakopoulou K, Samoli E. Air pollution and Parkinson's disease: A systematic review and meta-analysis up to 2018. Int J Hyg Environ Health. 2019;222:402-9. https://doi.org/10.1016/j.ijheh.2018.12.006.

28. Seelen M, Toro Campos RA, Veldink JH, Visser AE, Hoek G, Brunekreef B, et al. Long-Term Air Pollution Exposure and Amyotrophic Lateral Sclerosis in Netherlands: A Population-based Case-control Study. Environ Health Perspect. 2017;125:97023. https://doi.org/10.1289/EHP1115.

29. Rigolon A, Browning MHEM, McAnirlin O, Yoon H. Green Space and Health Equity: A Systematic Review on the Potential of Green Space to Reduce Health Disparities. Int J Environ Res Public Health. 2021;18:5. https://doi.org/10.3390/ijerph18052563.

30. Rodriguez-Loureiro L, Casas L, Bauwelinck M, Lefebvre W, Vanpoucke C, Vanroelen C, et al. Social inequalities in the associations between urban green spaces, self-perceived health and mortality in Brussels: Results from a census-based cohort study. Health Place. 2021;70:102603. https://doi.org/10.1016/j.healthplace.2021.102603.

31. Luyten S, Van Hecke E. Statistics Belgium Working Paper: De Belgische Stadsgewesten 2001 [in English: the Belgian city districts 2001] [Internet]. Statbel; 2007. Available from: https://statbel.fgov.be/sites/default/files/Over_Statbel_FR/Sociaal-Economische Enquête 2001 working paper - DE BELGISCHE STADSGEWESTEN.pdf (Accessed 03.02.2022).

32. World Health Organization (WHO). ICD-10 Version: 2016 [Online]. 2016. Available from: https://icd.who.int/browse10/2016/en (Accessed 03.02.2022).

33. Kramarow EA, Tejada-Vera B. Dementia Mortality in the United States, 2000-2017. Natl Vital Stat Reports. 2019;68:1-29.

34. Stokes AC, Weiss J, Lundberg DJ, Xie W, Kim JK, Preston SH, et al. Estimates of the Association of Dementia With US Mortality Levels Using Linked Survey and Mortality Records. JAMA Neurol. 2020;77:1543-50. https://doi.org/10.1001/jamaneurol.2020.2831.

35. Hobson P, Meara J. Mortality and quality of death certification in a cohort of patients with Parkinson's disease and matched controls in North Wales, UK at 18 years: a community-based cohort study. BMJ Open. 2018;8:e018969. https://doi.org/10.1136/bmjopen-2017-018969.

36. Bauwelinck M, Casas L, Nawrot TS, Nemery B, Trabelsi S, Thomas I, et al. Residing in urban areas with higher green space is associated with lower mortality risk: A census-based cohort study with ten years of follow-up. Environ Int. 2021;148:106365. https://doi.org/10.1016/j.envint.2020.106365.

37. Weier J, Herring D. Measuring Vegetation (NDVI \& EVI) [Online]. 2000. Available from: https://earthobservatory.nasa.gov/features/MeasuringVegetation (Accessed 03.02.2022).

38. Hooyberghs J, Mensink C, Dumont G, Fierens F. Spatial interpolation of ambient ozone concentrations from sparse monitoring points in Belgium. J Environ Monit. 2006;8:1129-35. https://doi.org/10.1039/b612607n.

39. Lefebvre W, Vranckx S. Validation of the IFDM-model for use in urban applications: study accomplished in the framework of the ATMOSYS-project. In: VITO Report 2013/RMA/R/56. VITO: Mol, Belgium; 2013. 
40. Falcaro M, Nur U, Rachet B, Carpenter JR. Estimating Excess Hazard Ratios and Net Survival When Covariate Data Are Missing: Strategies for Multiple Imputation. Epidemiology. 2015;26:421-8. https://doi.org/10.1097/EDE.0000000000000283.

41. Vienneau D, Hoogh K, De, Faeh D, Kaufmann M, Marc J, Röösli M, et al. More than clean air and tranquillity: Residential green is independently associated with decreasing mortality. Environ Int. 2017;108:176-84. http://dx.doi.org/10.1016/j.envint.2017.08.012.

42. van Buuren S. Flexible Imputation of Missing Data [Online]. Second Edi. Chapman\&Hall/CRC, editor. CRC Press, Taylor\&Francis Group; 2018. Available from: https://stefvanbuuren.name/fimd/ (Accessed 03.02.2022).

43. Klompmaker JO, Janssen NAH, Bloemsma LD, Gehring U, Wijga AH, Brink C, Van Den, et al. Associations of Combined Exposures to Surrounding Green, Air Pollution and Road Traffic Noise with Cardiometabolic Diseases. Environ Health Perspect. 2019;127:1-15.

44. Tingley D, Yamamoto T, Hirose K, Keele L, Imai K. mediation: R Package for Causal Mediation Analysis. J Stat Softw. 2014;59:1-38.

45. Imai K, Keele L, Tingley D. A General Approach to Causal Mediation Analysis. Psychol Methods. 2010;15:309-34.

46. R Core Team. R: A language and environment for statistical computing. Vienna: R Foundation for Statistical Computing; 2020.

47. van Buuren S, Groothuis-Oudshoorn K. mice: Multivariate Imputation by Chained Equations in R. J Stat Softw. 2011;45:1-67.

48. Therneau TM. _A Package for Survival Analysis in R_. R package version 3.2-11. 2021.

49. Wickham H. ggplot2: Elegant Graphics for Data Analysis. New York: Springer-Verlag; 2016.

50. VanderWeele TJ. Mediation Analysis: A Practitioner's Guide. Annu Rev Public Health. 2016;37:17-32. https://doi.org/10.1146/annurev-publhealth-032315-021402.

51. Singh-Manoux A, Dugravot A, Fournier A, Abell J, Ebmeier K, Kivimäki M, et al. Trajectories of Depressive Symptoms Before Diagnosis of Dementia: A 28-Year Follow-up Study. JAMA Psychiatry. 2017;74:712-8. https://doi.org/10.1001/jamapsychiatry.2017.0660.

52. Zotcheva E, Bergh S, Selbæk G, Krokstad S, Håberg AK, Strand BH, et al. Midlife Physical Activity, Psychological Distress, and Dementia Risk: The HUNT Study. J Alzheimer's Dis. 66: IOS Press; 2018. pp. 825-33.

53. Evans IEM, Martyr A, Collins R, Brayne C, Clare L. Social Isolation and Cognitive Function in Later Life: A Systematic Review and Meta-Analysis. J Alzheimer's Dis IOS Press. 2019;70:119-44.

54. Hong A, Sallis JF, King AC, Conway TL, Saelens B, Cain KL, et al. Linking green space to neighborhood social capital in older adults: The role of perceived safety. Soc Sci Med. 2018;207:38-45. https://doi.org/10.1016/j.socscimed.2018.04.051.

55. Astell-Burt T, Hartig T, Eckermann S, Nieuwenhuijsen M, McMunn A, Frumkin H, et al. More green, less lonely? A longitudinal cohort study. Int J Epidemiol. 2021. https://doi.org/10.1093/ije/dyab089. 
56. Markevych I, Schoierer J, Hartig T, Chudnovsky A, Hystad P, Dzhambov AM, et al. Exploring pathways linking greenspace to health: Theoretical and methodological guidance. Environ Res. 2017;158:30117. https://doi.org/10.1016/j.envres.2017.06.028.

57. Tallis M, Taylor G, Sinnett D, Freer-Smith P. Estimating the removal of atmospheric particulate polution by the urban tree canopy of London, under current and future environments. Landsc Urban Plan. 2011;103:129-38.

58. Vos PEJ, Maiheu B, Vankerkom J, Janssen S. Improving local air quality in cities: To tree or not to tree? Environ Pollut. 2013;183:113-22. https://doi.org/10.1016/j.envpol.2012.10.021.

59. Bowler DE, Buyung-ali L, Knight TM, Pullin AS. Urban greening to cool towns and cities: A systematic review of the empirical evidence. Landsc Urban Plan. 2010;97:147-55. http://dx.doi.org/10.1016/j.landurbplan.2010.05.006.

60. Watts N, Adger WN, Agnolucci P, Blackstock J, Byass P, Cai W, et al. Health and climate change: policy responses to protect public health. Lancet. 2015;386:1861-914. https://doi.org/10.1016/S01406736(15)60854-6.

61. Jitlal M, Amirthalingam GNK, Karania T, Parry E, Neligan A, Dobson R, et al. The Influence of Socioeconomic Deprivation on Dementia Mortality, Age at Death, and Quality of Diagnosis: A Nationwide Death Records Study in England and Wales 2001-2017. J Alzheimer's Dis. IOS Press; 2021;81:321-8.

62. Ascherio A, Schwarzschild MA. The epidemiology of Parkinson's disease: risk factors and prevention. Lancet Neuro. 2016;15:1257-72. https://doi.org/10.1016/S1474-4422(16)30230-7.

63. Brouwer M, Huss A, van der Mark M, Nijssen PCG, Mulleners WM, Sas AMG, et al. Environmental exposure to pesticides and the risk of Parkinson's disease in the Netherlands. Environ Int. 2017;107:100-10. https://doi.org/10.1016/j.envint.2017.07.001.

64. Majoka MA, Schimming C. Effect of Social Determinants of Health on Cognition and Risk of Alzheimer Disease and Related Dementias. Clin Ther. 2021. https://doi.org/10.1016/j.clinthera.2021.05.005.

65. Mitchell R, Popham F. Effect of exposure to natural environment on health inequalities: an observational population study. Lancet [Internet]. Elsevier Ltd; 2008;372:1655-60. Available from: http://dx.doi.org/10.1016/S0140-6736(08)61689-X.

66. KCE. Interventions pharmaceutiques et non pharmaceutiques dans la maladie d'Alzheimer: une évaluation rapide [in English: Pharmaceutical and non pharmaceutical interventions in Alzheimer's disease: a rapid evaluation] [Online]. Brussels, Belgium; 2009. Available from: https://kce.fgov.be/sites/default/files/atoms/files/d20091027328.pdf (Accessed 03.02.2022).

67. Deuschl G, Beghi E, Fazekas F, Varga T, Christoforidi KA, Sipido E, et al. The burden of neurological diseases in Europe: an analysis for the Global Burden of Disease Study 2017. Lancet Public Heal. 2020;5:e551-67. https://doi.org/10.1016/S2468-2667(20)30190-0.

68. World Health Organization (WHO). Global status report on the public health response to dementia [Online]. WHO: Geneva; 2021. Available from: 
https://www.who.int/publications/i/item/9789240033245 (Accessed 03.02.2022).

Figures

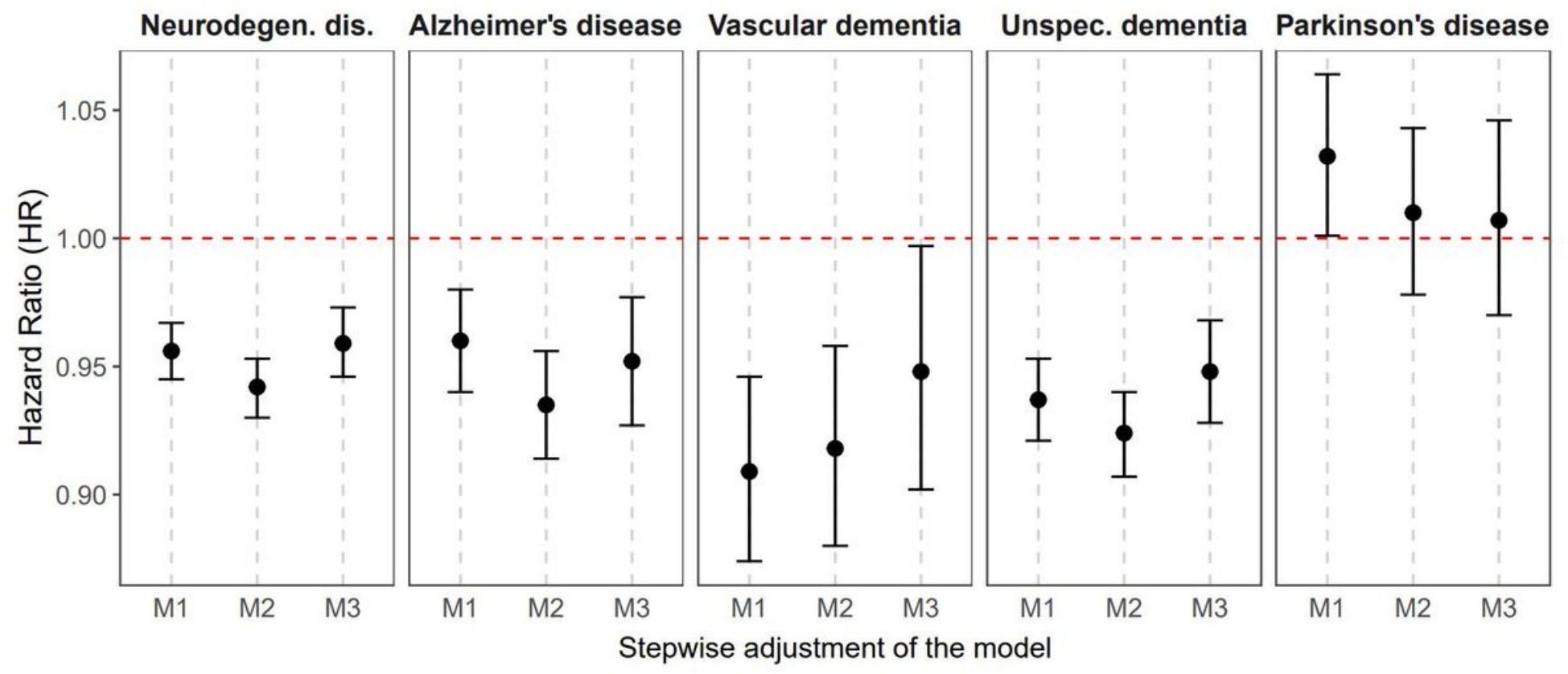

Figure 1

Associations (HRs) and 95\% $\mathrm{Cl}$ between residential surrounding greenness and overall and specific neurodegenerative disease mortality.

Five largest Belgian urban areas, 2001-2014. Results from Cox regression models using age as the underlying time scale. Model 1 included the baseline hazard, the strata terms for age and gender, and a frailty term for the urban areas; Model 2 added migrant background, household composition, educational level, and housing tenure; and Model 3 added median net taxable income at the statistical sector level. HRs expressed as one IQR increment (0.22) of residential surrounding greenness (buffer size 500-m) and neurodegenerative disease mortality outcomes. 


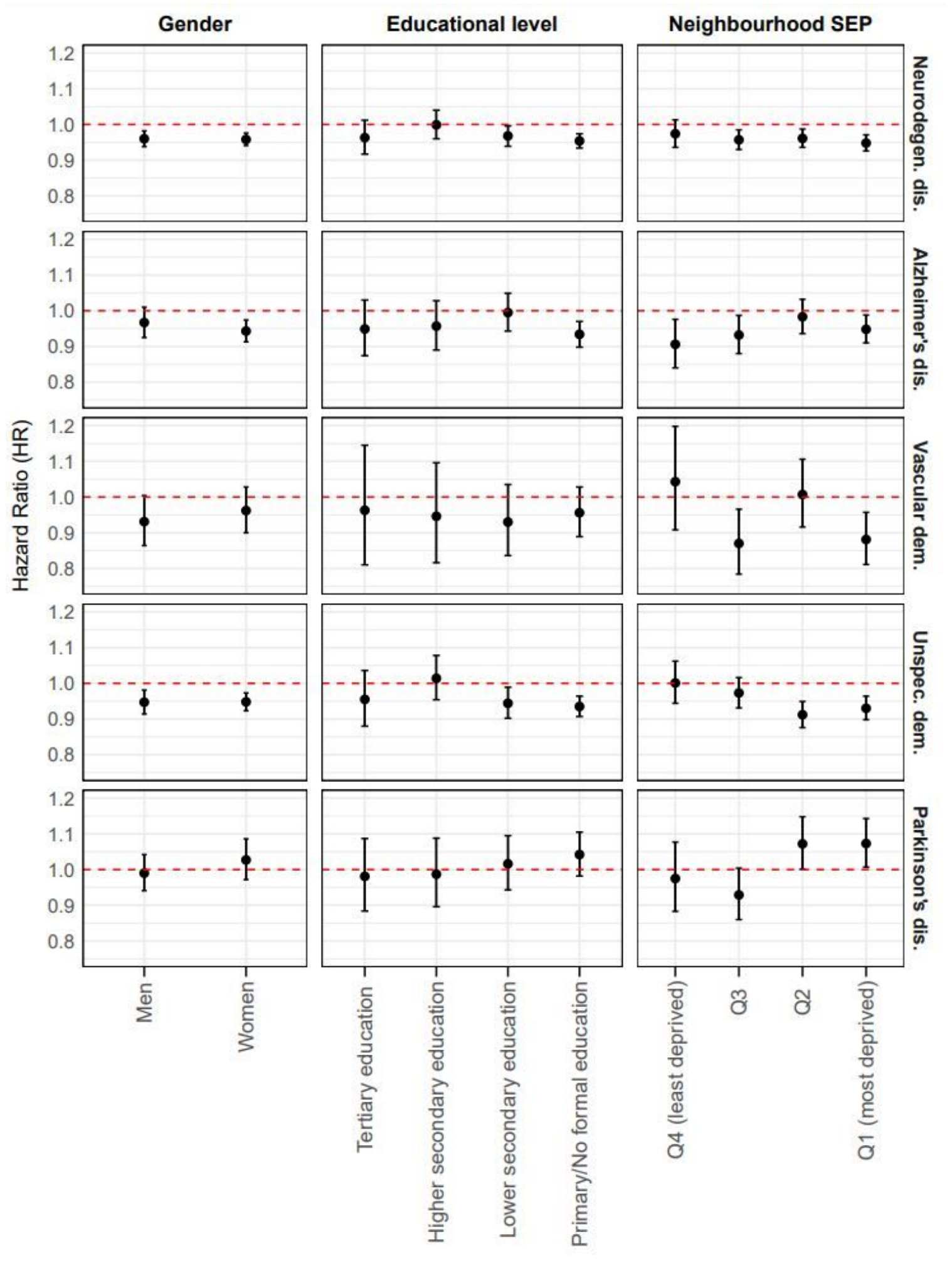

Figure 2

Associations (surrounding greenness and neurodegenerative disease mortality) stratified by gender, educational level and neighbourhood SEP.

Five largest Belgian urban areas, 2001-2014. Results from Cox regression models using age as the underlying time scale. Models included strata terms for age and gender, a frailty term for urban areas, and were adjusted for migrant background, household composition, educational level, housing tenure, and 
median net taxable income at the census tract level. Quartiles of exposure of area median net taxable income: Q1 (5,676-16,471], Q2 (16,471-19094], Q3 (19,094-21,091], Q4 (21,091-51,473].

\section{Supplementary Files}

This is a list of supplementary files associated with this preprint. Click to download.

- SupplementaryMaterial.docx 